\title{
A GEOMETRIC CLASSIFICATION OF IMMERSIONS OF 3-MANIFOLDS INTO 5-SPACE
}

\author{
ANDRÁS JUHÁSZ
}

\begin{abstract}
In this paper we define two regular homotopy invariants $c$ and $i$ for immersions of oriented 3-manifolds into $\mathbb{R}^{5}$ in a geometric manner. The pair $(c(f), i(f))$ completely describes the regular homotopy class of the immersion $f$. The invariant $i$ corresponds to the 3 -dimensional obstruction that arises from Hirsch-Smale theory and extends the one defined in [10 for immersions with trivial normal bundle.
\end{abstract}

\section{INTRODUCTION}

Hirsch-Smale theory [5] reduces the problem of regular homotopy classification of immersions to homotopy theory. However, this homotopy theoretic problem is usually hard to deal with. In the case of immersions of oriented 3-manifolds into $\mathbb{R}^{5}$ this homotopy theoretic problem was solved by Wu 14] using algebraic topological methods (also see [1).

However, it remains a problem to determine the regular homotopy class of a given immersion from its geometry. By geometry we mean the structure of the "singularities" of the map. For example, double points are such "singularities", and indeed, Smale [11] showed that for $n>1$ the regular homotopy class of an immersion $S^{n} \rightarrow \mathbb{R}^{2 n}$ is completely determined by the number of its double points (modulo 2 if $n$ is odd). A similar classification was carried out by Ekholm [2] for immersions of $S^{k}$ into $\mathbb{R}^{2 k-1}$ for $k \geq 4$.

The whole picture changes when we consider immersions of $S^{3}$ into $\mathbb{R}^{5}$. Hughes and Melvin [6] showed that there are infinitely many embeddings $S^{3} \hookrightarrow \mathbb{R}^{5}$ that are pairwise not regularly homotopic to each other. Therefore one can not determine the regular homotopy class from the "singularities" since an embedding has no such. Ekholm and Szücs [3 came over this problem using "singular Seifert surfaces" bounded by the immersions. For an immersion $f: M^{3} \rightarrow \mathbb{R}^{5}$ a singular Seifert surface is a generic map $F: W^{4} \rightarrow \mathbb{R}^{5}$ of a compact orientable manifold $W^{4}$ with boundary $M^{3}$ such that $\partial F=f$. In [3] it is shown that for $M^{3}=S^{3}$ the Smale invariant of $f$ can be computed from the singularities of $F$. Later Saeki, Szücs and Takase 10] generalized these results for immersions $f: M^{3} \rightarrow \mathbb{R}^{5}$ with trivial normal bundle (for oriented $M^{3}$ ). The invariant introduced in [10] corresponds to the 3dimensional obstruction to a regular homotopy between two such immersions. Our present paper generalizes the results of [10] to arbitrary immersions $f: M^{3} \rightarrow \mathbb{R}^{5}$.

We will consider the set $\operatorname{Imm}\left(M^{3}, \mathbb{R}^{5}\right)_{\chi}$ of immersions with fixed normal Euler class $e\left(\nu_{f}\right)=\chi \in H^{2}\left(M^{3} ; \mathbb{Z}\right)$ and construct a $\mathbb{Z}_{2 d(\chi)}$-valued regular homotopy

Date: November 13, 2018.

1991 Mathematics Subject Classification. 57N35; 57R45; 57R42.

Key words and phrases. immersion, regular homotopy, singular map, rotation. 
invariant $i$ for this set of immersions, where $d(\chi)$ denotes the divisibility of $\chi$. The construction of the invariant $i$ will also make use of a singular Seifert surface $F$. In [10] $F$ had to be an immersion near the boundary, but we (have to and) will allow arbitrary generic maps. If $\chi=0$ and $F$ is an immersion near the boundary then the construction of the invariant $i$ agrees with the one introduced in 10. We will also show that whenever $f, g: M^{3} \rightarrow \mathbb{R}^{5}$ are regularly homotopic on a neighborhood of the 2-skeleton of $M^{3}$ then $i(f)=i(g)$ iff $f$ and $g$ are regularly homotopic. This shows that $i$ corresponds to the 3 -dimensional obstruction to a regular homotopy between $f$ and $g$. (Note that there is an invariant which determines the regular homotopy class of the restriction of an immersion to a neighborhood of the 2skeleton of $M^{3}$. This invariant was called the $\mathrm{Wu}$ invariant in [10, see below.)

Regular homotopy classes of immersions of oriented 3-manifolds into $\mathbb{R}^{5}$ endowed with the connected sum operation form a semigroup whose structure we will also determine. Finally, an exact sequence will be defined that relates $\operatorname{Imm}\left[M^{3}, \mathbb{R}^{5}\right]$ to $\operatorname{Imm}\left[M^{3}, \mathbb{R}^{6}\right]$ and $\left[M^{3}, S^{2}\right]$.

\section{Preliminaries}

First let us recall the result of $\mathrm{Wu}$ [14 that classifies immersions of an oriented 3-manifold $M^{3}$ into $\mathbb{R}^{5}$ up to regular homotopy.

Theorem 2.1. The normal Euler class $\chi$ of an immersion $f: M^{3} \rightarrow \mathbb{R}^{5}$ is of the form $2 c$ for some $c \in H^{2}\left(M^{3} ; \mathbb{Z}\right)$ and for any $c \in H^{2}\left(M^{3} ; \mathbb{Z}\right)$ there is an immersion $f$ such that $\chi=2 c$. Furthermore,

$$
\operatorname{Imm}\left[M^{3}, \mathbb{R}^{5}\right]_{\chi} \approx \coprod_{c \in H^{2}\left(M^{3} ; \mathbb{Z}\right), 2 c=\chi} H^{3}\left(M^{3} ; \mathbb{Z}\right) /\left(2 \chi \cup H^{1}\left(M^{3} ; \mathbb{Z}\right)\right),
$$

where $\operatorname{Imm}\left[M^{3}, \mathbb{R}^{5}\right]_{\chi}$ is the set of regular homotopy classes of immersions with normal Euler class $\chi \in H^{2}\left(M^{3} ; \mathbb{Z}\right)$ and $\cup$ represents the cup product, moreover the symbol $\approx$ denotes a bijection.

Remark 2.2. For $\chi \in H^{2}\left(M^{3} ; \mathbb{Z}\right)$ let $d(\chi) \in \mathbb{Z}$ denote the divisibility of $\chi$, so that $\chi$ equals $d(\chi)$ times a primitive class in $H^{2}\left(M^{3} ; \mathbb{Z}\right)$ modulo torsion, and $d(\chi)=0$ if $\chi$ is of finite order. Then Poincaré duality implies that

$$
H^{3}\left(M^{3} ; \mathbb{Z}\right) /\left(2 \chi \cup H^{1}\left(M^{3} ; \mathbb{Z}\right)\right) \approx \mathbb{Z}_{2 d(\chi)} .
$$

If $f$ is an immersion of $M^{3}$ into $\mathbb{R}^{5}$ with normal Euler class $\chi$ then let us introduce the notation $d(f)$ for $d(\chi)$.

Notation 2.3. For $\chi \in H^{2}\left(M^{3} ; \mathbb{Z}\right)$ let $\Gamma_{2}(\chi)$ denote the set $\left\{c \in H^{2}\left(M^{3} ; \mathbb{Z}\right): 2 c=\right.$ $\chi\}$. Throughout this paper we will use the notation $M_{\circ}^{3}$ for the punctured 3manifold $M^{3} \backslash D^{3}$, where $D^{3} \subset M^{3}$ is a closed 3-disc. Then the 2-skeleton $\operatorname{sk}_{2}\left(M^{3}\right)$ is a deformation retract of $M_{\circ}^{3}$.

Theorem 2.1 can also be applied to the open manifold $M_{\circ}^{3}$. Since $H^{3}\left(M_{\circ}^{3} ; \mathbb{Z}\right)=0$ we obtain a bijection

$$
\bar{c}: \operatorname{Imm}\left[M_{\circ}^{3}, \mathbb{R}^{5}\right]_{\chi} \rightarrow \Gamma_{2}(\chi) .
$$

Thus for an immersion $f: M^{3} \rightarrow \mathbb{R}^{5}$ the invariant $c(f)=\bar{c}\left(f \mid M_{\circ}^{3}\right) \in \Gamma_{2}(\chi)$ describes the regular homotopy class of $f \mid M_{\circ}^{3}$. Following [10] we will call $c(f)$ the $\mathrm{Wu}$ invariant of the immersion $f$. 
To get a complete description of $\operatorname{Imm}\left[M^{3}, \mathbb{R}^{5}\right]_{\chi}$ we will construct a $\mathbb{Z}_{2 d(\chi)}$-valued invariant $i$ such that the map

$$
(c, i): \operatorname{Imm}\left[M^{3}, \mathbb{R}^{5}\right]_{\chi} \rightarrow \Gamma_{2}(\chi) \times \mathbb{Z}_{2 d(\chi)}
$$

will be a bijection. The invariant $i$ is constructed in a geometric manner and is an extension of the invariant defined in [10] for $\chi=0$.

Next let us recall Theorem 1.1(a) in [3]. Let $f: S^{3} \rightarrow \mathbb{R}^{5}$ be an immersion and $V^{4}$ an arbitrary compact oriented 4-manifold with $\partial V^{4}=S^{3}$. The map $f$ extends to a generic map $F: V^{4} \rightarrow \mathbb{R}^{5}$ which has no singular points near the boundary $\partial V^{4}$ since the normal bundle $\nu_{f}$ of $f$ is trivial. This map $F$ has isolated cusps, each one having a sign. Let us denote by $\# \Sigma^{1,1}(F)$ their algebraic number and let $\Omega(f)$ be the Smale invariant of $f$. The following formula was proved in [3].

\section{Theorem 2.4.}

$$
\Omega(f)=\frac{1}{2}\left(3 \sigma\left(V^{4}\right)+\# \Sigma^{1,1}(F)\right) .
$$

The proof of this theorem relies on the following proposition ([13], Lemma 3).

Lemma 2.5. Let $X^{4}$ be a closed oriented 4-manifold and $g: X^{4} \rightarrow \mathbb{R}^{5}$ a generic map. Then $3 \sigma\left(X^{4}\right)+\# \Sigma^{1,1}(g)=0$.

For the sake of completeness we will also recall from [10] the definition of the invariant $i$ for immersions with trivial normal bundle. First we need a preliminary definition.

Definition 2.6. Let $M^{3}$ be a closed oriented 3-manifold. We denote by $\alpha\left(M^{3}\right)$ the dimension of the $\mathbb{Z}_{2}$ vector space $\tau H_{1}\left(M^{3} ; \mathbb{Z}\right) \otimes \mathbb{Z}_{2}$, where $\tau H_{1}\left(M^{3} ; \mathbb{Z}\right)$ is the torsion subgroup of $H_{1}\left(M^{3} ; \mathbb{Z}\right)$.

Definition 2.7. Let $f: M^{3} \rightarrow \mathbb{R}^{5}$ be an immersion with trivial normal bundle. Let $W^{4}$ be any compact oriented 4-manifold with $\partial W^{4}=M^{3}$ and $F: W^{4} \rightarrow \mathbb{R}^{5}$ a generic map nonsingular near the boundary such that $F \mid \partial W^{4}=f$. (We can choose such a generic map $F$ since $f$ is an immersion with trivial normal bundle.) Denote the algebraic number of cusps of $F$ by $\# \Sigma^{1,1}(F)$. Then let

$$
i(f)=\frac{3}{2}\left(\sigma\left(W^{4}\right)-\alpha\left(M^{3}\right)\right)+\frac{1}{2} \# \Sigma^{1,1}(F) .
$$

It is proved in 10] that $i(f)$ is always an integer and a regular homotopy invariant.

In the following sections we will extend the above regular homotopy invariant $i$ to arbitrary immersions. If $f: M^{3} \rightarrow \mathbb{R}^{5}$ has non-trivial normal bundle then we have to give up the assumption that the singular Seifert-surface $F$ is an immersion near the boundary. Thus we will use an arbitrary generic map $F: W^{4} \rightarrow \mathbb{R}^{5}$ such that $\partial F=f$. The singular set $\Sigma^{1}(F)$ of such an $F$ is a 2-dimensional submanifold of $W^{4}$ with boundary $C(F)=\partial \Sigma^{1}(F) \subset M^{3}$. If we orient $\operatorname{ker}(d F) \mid C(F)$ so that it points into $W^{4}$ and project it into $T M^{3}$ then we obtain a normal field $\nu(F)$ along $C(F)$. We will define the rotation of $\nu(F)$ around $C(F)$ modulo $4 d(f)$ and denote this by $R(F)$. The double of the extended invariant will be defined to be

$$
I(f)=3\left(\sigma\left(W^{4}\right)-\alpha\left(M^{3}\right)\right)+\# \Sigma^{1,1}(F)+R(F) \in \mathbb{Z}_{4 d(f)} .
$$

We will show that $I(f)$ is always even, thus it defines an element $i(f) \in \mathbb{Z}_{2 d(f)}$ using the natural embedding $\mathbb{Z}_{2 d(f)} \hookrightarrow \mathbb{Z}_{4 d(f)}$. 


\section{Rotation}

Throughout this paper $M^{3}$ will denote a fixed closed connected and oriented 3-manifold.

Notation 3.1. A pair $(C, \nu)$ will always stand for an oriented 1-dimensional submanifold $C$ of $M^{3}$ and a nowhere vanishing normal field $\nu$ along $C$.

Definition 3.2. Let $\chi \in H^{2}\left(M^{3} ; \mathbb{Z}\right)$ and let $C_{0}$ and $C_{1}$ be 1-dimensional oriented submanifolds of $M^{3}$ with normal fields $\nu_{0}$ and $\nu_{1}$ such that $P D\left[C_{0}\right]=P D\left[C_{1}\right]=\chi$. (Here $P D$ denotes Poincaré duality.) Then we can define the rotation difference $\operatorname{rd}\left(\left(C_{0}, \nu_{0}\right),\left(C_{1}, \nu_{1}\right)\right) \in \mathbb{Z}_{2 d(\chi)}$ of $\left(C_{0}, \nu_{0}\right)$ and $\left(C_{1}, \nu_{1}\right)$ as follows. Since $\left[C_{0}\right]=\left[C_{1}\right]$ and

$$
H_{1}\left(M^{3} ; \mathbb{Z}\right) \approx H^{2}\left(M^{3} ; \mathbb{Z}\right) \approx\left[M^{3}, \mathbb{C} P^{\infty}\right],
$$

there exists an oriented cobordism $K^{2} \subset M^{3} \times I$ between $C_{0} \subset M^{3} \times\{0\}$ and $C_{1} \subset M^{3} \times\{1\}$. Let $\nu$ be a generic normal field along $K^{2}$ that extends $\nu_{0}$ and $\nu_{1}$. Then a sign can be given to each zero of $\nu$ since $M^{3}$ is oriented. Now we define $\operatorname{rd}\left(\left(C_{0}, \nu_{0}\right),\left(C_{1}, \nu_{1}\right)\right)$ to be the algebraic number of zeroes of $\nu$ modulo $2 d(\chi)$. Equivalently, $\operatorname{rd}\left(\left(C_{0}, \nu_{0}\right),\left(C_{1}, \nu_{1}\right)\right)$ is the self intersection of $K$ in $M^{3} \times I$ modulo $2 d(\chi)$ if perturbed in the direction of $\nu$.

Remark 3.3. The rotation difference is the obstruction to the existence of a framed cobordism between the framed submanifolds $\left(C_{0}, \nu_{0}\right)$ and $\left(C_{1}, \nu_{1}\right)$ of $M^{3}$. Using the Pontrjagin construction this corresponds to the obstruction to a homotopy between two maps of $M^{3}$ to $S^{2}$. This situation was first examined in [9]. It is easy to see that $\operatorname{rd}\left(\left(C_{0}, \nu_{0}\right),\left(C_{1}, \nu_{1}\right)\right)=0$ iff $\left(C_{0}, \nu_{0}\right)$ and $\left(C_{1}, \nu_{1}\right)$ are framed cobordant. Thus we obtain a bijection

$$
\left[M^{3}, S^{2}\right] \approx \coprod_{\chi \in H^{2}\left(M^{3} ; \mathbb{Z}\right)} H^{3}\left(M^{3} ; \mathbb{Z}\right) / 2 \chi \cup H^{1}\left(M^{3} ; \mathbb{Z}\right) .
$$

Proposition 3.4. In Definition 3.2 above the rotation difference is well defined, i.e., it does not depend on the choice of $K$ and $\nu$.

Proof. Let $K, \nu$ and $K^{\prime}, \nu^{\prime}$ be as in Definition [3.2 We glue together $M^{3} \times I$ and $-M^{3} \times I$ along their boundaries so that we obtain the double $D\left(M^{3} \times I\right)=M^{3} \times S^{1}$. Place $K$ into the half of $M^{3} \times S^{1}$ corresponding to $M^{3} \times I$ and $K^{\prime}$ into the other half. Then we obtain a closed oriented surface $F=K \cup-K^{\prime}$ in $M^{3} \times S^{1}$ and a normal field $\mu=\nu \cup \nu^{\prime}$ along $F$. Since $H^{*}\left(S^{1} ; \mathbb{Z}\right)$ is a torsion free $\mathbb{Z}$-module we can apply Künneth's theorem and we get that

$$
H^{2}\left(M^{3} \times S^{1} ; \mathbb{Z}\right) \approx H^{1}\left(M^{3} ; \mathbb{Z}\right) \otimes H^{1}\left(S^{1} ; \mathbb{Z}\right) \oplus H^{2}\left(M^{3} ; \mathbb{Z}\right) \otimes H^{0}\left(S^{1} ; \mathbb{Z}\right)
$$

Thus the Poincaré dual of $F$ can be written in the form

$$
P D[F]=x \times \alpha+y \times 1 \in H^{2}\left(M^{3} \times S^{1} ; \mathbb{Z}\right),
$$

where $x \in H^{1}\left(M^{3} ; \mathbb{Z}\right)$ and $y \in H^{2}\left(M^{3} ; \mathbb{Z}\right)$, moreover $\alpha$ denotes the generator of $H^{1}\left(S^{1} ; \mathbb{Z}\right)$ and 1 the generator of $H^{0}\left(S^{1} ; \mathbb{Z}\right)$ given by the orientation of $S^{1}$. Note that for $1 \in S^{1}$ the dual class of $M^{3} \times\{1\} \subset M^{3} \times S^{1}$ is

$$
P D\left[M^{3} \times\{1\}\right]=P D\left[M^{3}\right] \times P D[\{1\}]=1 \times \alpha \in H^{1}\left(M^{3} \times S^{1} ; \mathbb{Z}\right) .
$$

Moreover,

$$
P D\left[F \cap\left(M^{3} \times\{1\}\right)\right]=P D\left[C_{1} \times\{1\}\right]=P D\left[C_{1}\right] \times P D[\{1\}]=\chi \times \alpha .
$$


On the other hand

$P D\left[F \cap\left(M^{3} \times\{1\}\right)\right]=P D[F] \cup P D\left[M^{3} \times\{1\}\right]=(x \times \alpha+y \times 1) \cup(1 \times \alpha)=x \times \alpha^{2}+y \times \alpha$.

Since $\alpha^{2}=0$ we get that $y \times \alpha=\chi \times \alpha$. Using Künneth's theorem again we obtain the equality $y=\chi$. Thus we get that

$$
P D[F] \cup P D[F]=(x \times \alpha+\chi \times 1)^{2}=(2 x \cup \chi) \times \alpha
$$

since $\alpha^{2}=\chi^{2}=0$ and $x \cup \chi=\chi \cup x$ because the degree of $\chi$ is 2 . So the self intersection of $F$ in $M^{3} \times S^{1}$ equals $\left\langle(2 \chi \cup x) \times \alpha,\left[M^{3} \times S^{1}\right]\right\rangle=\left\langle 2 \chi \cup x,\left[M^{3}\right]\right\rangle \in$ $2 d(\chi) \mathbb{Z}$. If we perturb $F$ in the direction of $\mu$ we get that the self intersection of $K$ with respect to $\nu$ equals the self intersection of $K^{\prime}$ with respect to $\nu^{\prime}$ modulo $2 d(\chi)$.

Proposition 3.5. If $\left[C_{0}\right]=\left[C_{1}\right]=\left[C_{2}\right] \in H_{1}\left(M^{3}, \mathbb{Z}\right)$ then

$$
r d\left(\left(C_{0}, \nu_{0}\right),\left(C_{1}, \nu_{1}\right)\right)+r d\left(\left(C_{1}, \nu_{1}\right),\left(C_{2}, \nu_{2}\right)\right)=r d\left(\left(C_{0}, \nu_{0}\right),\left(C_{2}, \nu_{2}\right)\right) .
$$

Definition 3.6. For each $a \in H_{1}\left(M^{3} ; \mathbb{Z}\right)$ fix a pair $\left(C_{a}, \nu_{a}\right)$ such that $\left[C_{a}\right]=a$. Then for $[C]=a$ let $r(C, \nu)=\operatorname{rd}\left((C, \nu),\left(C_{a}, \nu_{a}\right)\right)$.

Corollary 3.7. If $\left[C_{0}\right]=\left[C_{1}\right]$ then $r\left(C_{0}, \nu_{0}\right)-r\left(C_{1}, \nu_{1}\right)=r d\left(\left(C_{0}, \nu_{0}\right),\left(C_{1}, \nu_{1}\right)\right)$.

Definition 3.8. We can define the mod 2 rotation difference $\operatorname{rd}_{2}\left(\left(C_{0}, \nu_{0}\right),\left(C_{1}, \nu_{1}\right)\right)$ for unoriented $C_{0}$ and $C_{1}$ just as in Definition 3.2 but allowing the cobordism $K$ to be non-orientable and counting the self intersection of $K$ in $M \times I$ only modulo 2 . The proof that this is well defined is analogous to the oriented case. It is clear that the epimorphism $\mathbb{Z}_{2 d(\chi)} \rightarrow \mathbb{Z}_{2}$ takes rd to $\operatorname{rd}_{2}$. The mod 2 rotation $r_{2}$ is defined just like $r$.

Unfortunately we will have to lift the invariants rd and $r$ to $\mathbb{Z}_{4 d(\chi)}$. To be able to do this we need more structure on $M^{3}$ then just a framed submanifold. We will use this additional structure to restrict the homology class of the cobordism $K$ so that the surface $F$ in the proof of Proposition 3.4 will represent an even homology class and thus $x$ will always be even (since $\chi$ is even). So the self intersection of $F$ will be divisible by $4 d(\chi)$ instead of just $2 d(\chi)$.

Notation 3.9. Fix a cohomology class $\chi \in H^{2}\left(M^{3} ; \mathbb{Z}\right)$. Let $\varepsilon_{M}^{3}$ denote the 3 dimensional trivial bundle over $M^{3}$ and let $t, v \in \Gamma\left(\varepsilon_{M}^{3}\right)$ be two generic non-zero sections of $\varepsilon_{M}^{3}$. Furthermore, suppose that the 2-dimensional oriented subbundle $t^{\perp}<\varepsilon_{M}^{3}$ has Euler class $\chi$. If we project $v$ into $t^{\perp}$ we obtain a section $w \in \Gamma\left(t^{\perp}\right)$ that vanishes along a curve $C \subset M^{3}$ and we orient $C$ so that $P D[C]=e\left(t^{\perp}\right)$. In particular, $t$ and $v$ are linearly dependent exactly at the points of $C$. Finally let $\nu$ be a non-zero normal field along $C$. In the future we will denote such a structure on $M^{3}$ by a quadruple $(C, \nu, t, v)$ and the set of these structures by $N\left(M^{3}, \chi\right)$.

Remark 3.10. Since $\left.P D[C]\right|_{2}=w_{2}\left(\varepsilon_{M}^{3}\right)=0 \in H^{2}\left(M^{3} ; \mathbb{Z}_{2}\right)$, the cohomology class $\chi=P D[C]$ is of the form $2 c$ for some $c \in H^{2}\left(M^{3} ; \mathbb{Z}\right)$. This can be seen from the long exact sequence associated to the coefficient sequence $\mathbb{Z} \rightarrow \mathbb{Z} \rightarrow \mathbb{Z}_{2}$. Thus $N\left(M^{3}, \chi\right)=\emptyset$ if $\chi$ is not of the form $2 c$.

Definition 3.11. Suppose that $a_{0}=\left(C_{0}, \nu_{0}, t_{0}, v_{0}\right)$ and $a_{1}=\left(C_{1}, \nu_{1}, t_{1}, v_{1}\right)$ are elements of $N\left(M^{3}, \chi\right)$, where $\chi=2 c$. Then we will define their rotation difference $\operatorname{Rd}\left(a_{0}, a_{1}\right) \in \mathbb{Z}_{4 d(\chi)}$ as follows. We will consider $a_{i}$ to be in $N\left(M^{3} \times\{i\}, \chi\right)$ for $i=0,1$. Let $t, v \in \Gamma\left(\varepsilon_{M \times I}^{3}\right)$ be generic non-zero sections extending $t_{i}$ and $v_{i}$ for 
$i=0,1$. Denote by $K$ the 2-dimensional submanifold of $M^{3} \times I$ where $t$ and $v$ are linearly dependent. Let $w$ denote the projection of $v$ into the 2-dimensional oriented subbundle $t^{\perp}<\varepsilon_{M \times I}^{3}$. Then $w$ is zero exactly at the points of $K$, thus it defines an orientation of $K$. With this orientation $K$ is an oriented cobordism between $C_{0}$ and $C_{1}$. Let $\nu$ denote a normal field of $K$ that extends both $\nu_{0}$ and $\nu_{1}$. Now we define $\operatorname{Rd}\left(a_{0}, a_{1}\right)$ to be the algebraic number of zeroes of $\nu$ modulo $4 d(\chi)$. Equivalently, $\operatorname{Rd}\left(a_{0}, a_{1}\right)$ is the self intersection of $K$ in $M^{3} \times I$ modulo $4 d(\chi)$ if perturbed in the direction of $\nu$.

Proposition 3.12. In Definition 3.11 the rotation difference is well defined. I.e., it does not depend on the extensions $t, v$ and $\nu$.

Proof. Let $t, v, \nu$ and $t^{\prime}, v^{\prime}, \nu^{\prime}$ be as in Definition 3.11 The sections $t, v$ are linearly dependent over $K$ and $t^{\prime}, v^{\prime}$ are dependent over $K^{\prime}$. Just as in the proof of Proposition 3.4 we will place $K, \nu$ and $K^{\prime}, \nu^{\prime}$ in the two halves of the double $D\left(M^{3} \times I\right)=M^{3} \times S^{1}$ and place $t, v$ and $t^{\prime}, v^{\prime}$ in the two halves of the trivial bundle $\varepsilon_{M \times S^{1}}^{3}$. Let $F$ denote the oriented surface $K \cup-K^{\prime}$ and by $\mu$ the normal field along $F$ obtained from $\nu$ and $\nu^{\prime}$. Moreover, let $T=t \cup t^{\prime}$ and $V=v \cup v^{\prime}$. Then $T, V \in \Gamma\left(\varepsilon_{M \times S^{1}}^{3}\right)$ are linearly dependent exactly over $F$, thus $\left.P D[F]\right|_{2}=w_{2}\left(\varepsilon_{M \times S^{1}}^{3}\right)=0 \in H^{2}\left(M^{3} \times S^{1} ; \mathbb{Z}_{2}\right)$. Using the coefficient sequence $\mathbb{Z} \rightarrow \mathbb{Z} \rightarrow \mathbb{Z}_{2}$ we get that $P D[F]$ is of the for $2 b$ for some $b \in H^{2}\left(M^{3} \times S^{1} ; \mathbb{Z}\right)$. Since $P D[F]$ is of the form $x \times \alpha+\chi \times 1$ where $\chi=2 c$ we get that there exists an element $z \in H^{1}\left(M^{3} ; \mathbb{Z}\right)$ such that $x=2 z$. Thus $P D[F]^{2}=(4 z \cup \chi) \times \alpha$ which implies that the self intersection of $F$ is divisible by $4 d(\chi)$. If we perturb $F$ in the direction of $\mu$ we get that the self intersection of $K$ with respect to $\nu$ equals the self intersection of $K^{\prime}$ with respect to $\nu^{\prime}$ modulo $4 d(\chi)$.

Remark 3.13. The surface $K$ represents the dual of the Stiefel-Whitney class of the bundle $\varepsilon_{M \times I}^{3}$ relative to the sections $t_{i}, v_{i}$ given over $M^{3} \times\{0,1\}$. I.e.,

$\left.P D[K]\right|_{2}=w_{2}\left(\varepsilon_{M \times I}^{3} ; t_{i}, v_{i}\right) \in H^{2}\left(M^{3} \times I,\left(\left(M^{3} \backslash C_{0}\right) \times\{0\}\right) \cup\left(\left(M^{3} \backslash C_{1}\right) \times\{1\}\right) ; \mathbb{Z}_{2}\right)$

since $v$ and $t$ are linearly independent over $\left(\left(M^{3} \backslash C_{0}\right) \times\{0\}\right) \cup\left(\left(M^{3} \backslash C_{1}\right) \times\{1\}\right)$. Using Lefschetz duality we get that the relative homology class $\left.[K]\right|_{2} \in H^{2}\left(M^{3} \times\right.$ $\left.I, C_{0} \times\{0\} \cup C_{1} \times\{1\} ; \mathbb{Z}_{2}\right)$ is independent of the choice of $t$ and $v$. If we choose a simplicial subdivision of $M^{3}$ so that $\operatorname{sk}_{1}\left(M^{3}\right) \cap C_{i}=\emptyset$ for $i=0,1$ then $w_{2}$ is the obstruction to extending the map $\left(t_{i}, v_{i}\right): \operatorname{sk}_{1}\left(M^{3} \times\{0,1\}\right) \rightarrow V_{2}\left(\mathbb{R}^{3}\right)$ to $\operatorname{sk}_{2}\left(M^{3} \times I\right)$. So the homology class $\left.[K]\right|_{2}$ and thus $\operatorname{Rd}\left(a_{0}, a_{1}\right)$ depends only on the homotopy class of the map

$$
\left(t_{i}, v_{i}\right) \mid \operatorname{sk}_{1}\left(M^{3}\right): \operatorname{sk}_{1}\left(M^{3}\right) \rightarrow V_{2}\left(\mathbb{R}^{3}\right)
$$

for $i=0,1$. For the sake of completeness we note that if the extension $t$ is given then

$$
P D[K]=e\left(t^{\perp} ; w_{i}\right) \in H^{2}\left(M^{3} \times I,\left(M^{3} \backslash C_{0}\right) \times\{0\} \cup\left(M^{3} \backslash C_{1}\right) \times\{1\} ; \mathbb{Z}\right) .
$$

So we have obtained the following proposition.

Proposition 3.14. Suppose that $\left(C_{0}, \nu_{0}\right)$ and $\left(C_{1}, \nu_{1}\right)$ are framed submanifolds of $M^{3}$ and let $a_{0}, b_{0}, a_{1}, b_{1} \in N\left(M^{3}, \chi\right)$ be of the form $a_{i}=\left(C_{i}, \nu_{i}, t_{i}^{a}, v_{i}^{a}\right)$ and $b_{i}=\left(C_{i}, \nu_{i}, t_{i}^{b}, v_{i}^{b}\right)$ for $i=0,1$. Moreover, suppose that $s_{1}\left(M^{3}\right) \cap C_{i}=\emptyset$ and 
$\left(t_{i}^{a}, v_{i}^{a}\right) \mid s k_{1}\left(M^{3}\right)$ is homotopic to $\left(t_{i}^{b}, v_{i}^{b}\right) \mid s k_{1}\left(M^{3}\right)$ as maps into $V_{2}\left(\mathbb{R}^{3}\right)$ for $i=0,1$. Then the following equality holds:

$$
R d\left(a_{0}, a_{1}\right)=\operatorname{Rd}\left(b_{0}, b_{1}\right) .
$$

Proof. Let $t^{a}$ and $v^{a}$ be generic extensions of $t_{i}^{a}$, respectively $v_{i}^{a}$ over $M^{3} \times I$ and denote by $K^{a}$ the submanifold of $M^{3} \times I$ where $t^{a}$ and $v^{a}$ are linearly dependent. We obtain the sections $t^{b}$ and $v^{b}$ of $\varepsilon_{M \times I}^{3}$ and the submanifold $K^{b} \subset M^{3} \times I$ in a similar way. Then, according to Remark 3.13 we get that

$$
\left.P D\left[K^{a}\right]\right|_{2}=w_{2}\left(\varepsilon_{M \times I}^{3} ; t_{i}^{a}, v_{i}^{a}\right)=w_{2}\left(\varepsilon_{M \times I}^{3} ; t_{i}^{b}, v_{i}^{b}\right)=\left.P D\left[K^{b}\right]\right|_{2},
$$

since $w_{2}$ is the obstruction to extending a map into $V_{2}\left(\mathbb{R}^{3}\right)$ from $\operatorname{sk}_{1}\left(M^{3} \times I\right)$ to $\operatorname{sk}_{2}\left(M^{3} \times I\right)$ and $\left(t_{i}^{a}, v_{i}^{a}\right) \mid \operatorname{sk}_{1}\left(M^{3} \times\{i\}\right)$ is homotopic to $\left(t_{i}^{b}, v_{i}^{b}\right) \mid \operatorname{sk}_{1}\left(M^{3} \times\{i\}\right)$. Thus if $F$ denotes the submanifold of $M^{3} \times S^{1}=D\left(M^{3} \times I\right)$ obtained by piecing together $K^{a}$ and $K^{b}$ we get that $\left.P D[F]\right|_{2}=w_{2}\left(\varepsilon_{M \times S^{1}}^{3}\right)=0$, so we can proceed as in the proof of Proposition 3.12

Proposition 3.15. If $a_{i}=\left(C_{i}, \nu_{i}, t_{i}, v_{i}\right) \in N\left(M^{3}, \chi\right)$ for $i=0,1$ then

$$
\operatorname{rd}\left(\left(C_{0}, \nu_{0}\right),\left(C_{1}, \nu_{1}\right)\right) \equiv \operatorname{Rd}\left(a_{0}, a_{1}\right) \bmod 2 d(\chi) .
$$

Proposition 3.16. If $a_{0}, a_{1}, a_{2} \in N\left(M^{3}, \chi\right)$ then

$$
R d\left(a_{0}, a_{1}\right)+\operatorname{Rd}\left(a_{1}, a_{2}\right)=\operatorname{Rd}\left(a_{0}, a_{2}\right) .
$$

Definition 3.17. For each $\chi \in H^{2}\left(M^{3} ; \mathbb{Z}\right)$ of the form $\chi=2 c$ fix an element $a_{\chi} \in N\left(M^{3}, \chi\right)$. Then for each $a \in N\left(M^{3}, \chi\right)$ define the dotation $R(a) \in \mathbb{Z}_{4 d(\chi)}$ to be $\operatorname{Rd}\left(a, a_{\chi}\right)$.

Corollary 3.18. If $a_{0}, a_{1} \in N\left(M^{3}, \chi\right)$ then $R d\left(a_{0}, a_{1}\right)=R\left(a_{0}\right)-R\left(a_{1}\right)$.

\section{THE ORIENTATION OF $\Sigma^{1}$}

Now let us recall a special case of Lemma 6.1 of 3 . Let $F: W^{4} \rightarrow \mathbb{R}^{5}$ be a generic map of a compact orientable manifold. Then the singularity set $\Sigma(F)$ of $F$ is a 2-dimensional submanifold of $W^{4}$ which is not necessarily orientable.

Lemma 4.1. The line bundles $\operatorname{det}(T \Sigma(F))$ and $\operatorname{ker}(d F)$ over $\Sigma(F)$ are isomorphic.

Definition 4.2. Let $\pi$ denote the projection of $\mathbb{R}^{m+1}$ onto $\mathbb{R}^{m}$. A map $f: N^{n} \rightarrow$ $\mathbb{R}^{m}$ is called prim if there exists an immersion $f^{\prime}: N^{n} \leftrightarrow \mathbb{R}^{m+1}$ such that $\pi \circ f^{\prime}=f$.

Corollary 4.3. If $F: W^{4} \rightarrow \mathbb{R}^{5}$ is a generic prim map then $\Sigma(F) \subset W^{4}$ is an orientable surface.

Proof. Let $s$ denote the sixth coordinate function of $F^{\prime}$, i.e., $F^{\prime}=(F, s)$. Since $F^{\prime}$ is non-singular, the function $s$ is non-degenerate along $\operatorname{ker}(d F)$. Thus we can orient $\operatorname{ker}(d F)$ so that the derivative of $s$ in the positive direction of $\operatorname{ker}(d F)$ is positive. But the orientability of $\operatorname{ker}(d F)$ implies the orientability of $\Sigma(F)$ by Lemma 4.1

The following definition, motivated by Corollary 4.3 gives an explicit isomorphism $\Psi$ between $\operatorname{ker}(d F)$ and $\operatorname{det}(T \Sigma(F))$.

Definition 4.4. Let $W^{4}$ be a compact oriented manifold with possibly non-empty boundary and let $F: W^{4} \rightarrow \mathbb{R}^{5}$ be a generic map. For $p \in \Sigma(F)$ choose a small neighborhood $U_{p} \subset W^{4}$ of $p$ in which $\operatorname{ker}(d F)$ is orientable. Put $F_{p}=F \mid U_{p}$ and choose an orientation $o_{p}$ of $\operatorname{ker}\left(d F_{p}\right)$. Then there exists a smooth function 
$s: U_{p} \rightarrow \mathbb{R}$ such that the derivative of $s$ in the direction of $o_{p}$ is positive. (First construct $s$ along $\Sigma\left(F_{p}\right)$ near $\Sigma^{1,1}\left(F_{p}\right)$ then extend it to a tubular neighborhood of $\Sigma\left(F_{p}\right)$.) The map $F_{p}^{\prime}=\left(F_{p}, s\right): U_{p} \rightarrow \mathbb{R}^{6}$ is an immersion. If $U_{p}$ is chosen sufficiently small then we can even suppose that $F_{p}^{\prime}$ is an embedding. Denote by $e_{6}$ the sixth coordinate direction in $\mathbb{R}^{6}$ and let $\nu_{6}: U_{p} \rightarrow T \mathbb{R}^{6}$ denote the vector field along $F_{p}^{\prime}$ defined by the formula $\nu_{6}(x)=e_{6} \in T_{F_{p}^{\prime}(x)} \mathbb{R}^{6}$ for $x \in U_{p}$. Projecting $\nu_{6}$ into the normal bundle of $F_{p}^{\prime}$ we obtain a normal field $\mu_{6}$ along $F_{p}^{\prime}$ that vanishes exactly at the points of $\Sigma\left(F_{p}\right)$. Perturb $F_{p}^{\prime}$ in the direction of $\mu_{6}$ to obtain an embedding $F_{p}^{\prime \prime}$. Then orient $\Sigma\left(F_{p}\right)$ as the intersection of $F_{p}^{\prime}$ and $F_{p}^{\prime \prime}$ in $\mathbb{R}^{6}$. Here $\mathbb{R}^{6}$ is considered with its standard orientation. This orientation of $\Sigma\left(F_{p}\right)$ does not depend on the choice of the function $s$, since if $s_{1}$ and $s_{2}$ are two such functions then for $0 \leq t \leq 1$ the convex combination $(1-t) s_{1}+t s_{2}$ also satisfies the conditions for $s$.

If we reverse the orientation of $\operatorname{ker}\left(d F_{p}\right)$, i.e. if we orient it by $-o_{p}$, then we can choose $-s$ instead of $s$. Thus we obtain the embedding $\left(F_{p},-s\right)$, which is the reflection of $F_{p}^{\prime}$ in the hyperplane $\mathbb{R}^{5}$. Denote this reflection by $R: \mathbb{R}^{6} \rightarrow \mathbb{R}^{6}$ (i.e., $\left.R\left(x_{1}, \ldots, x_{5}, x_{6}\right)=\left(x_{1}, \ldots, x_{5},-x_{6}\right)\right)$. Then $\left(F_{p},-s\right)=R \circ F_{p}^{\prime}$. The vector field $d R \circ \nu_{6}$ along $R \circ F_{p}^{\prime}$ points in the direction $-e_{6}$ and $R \circ F_{p}^{\prime \prime}$ is the perturbation of $R \circ F_{p}^{\prime}$ in the direction of $d R \circ \mu_{6}$. But in this case we should perturb $R \circ F_{p}^{\prime}$ in the direction of $-\left(d R \circ \mu_{6}\right)$. We obtain the same orientation if we look at the intersection $\left(R \circ F_{p}^{\prime \prime}\right) \cap\left(R \circ F_{p}^{\prime}\right)$ instead. Since the intersection is 2-dimensional and $U_{p}$ is 4-dimensional we get that $\left(R \circ F_{p}^{\prime \prime}\right) \cap\left(R \circ F_{p}^{\prime}\right)=\left(R \circ F_{p}^{\prime}\right) \cap\left(R \circ F_{p}^{\prime \prime}\right)$ in the oriented sense. The orientations of $\Sigma\left(F_{p}\right)$ defined by the intersections $F_{p}^{\prime} \cap F_{p}^{\prime \prime}$ and $\left(R \circ F_{p}^{\prime}\right) \cap\left(R \circ F_{p}^{\prime \prime}\right)$ are opposite. This can be seen from the following argument: For $0 \leq t \leq 1$ denote by $R_{t}$ the rotation of the hyperplane $\mathbb{R}^{6}$ in $\mathbb{R}^{7}$ around $\mathbb{R}^{5}$ by the angle $\pi t$. The orientation of $\left(R_{t} \circ F_{p}^{\prime}\right) \cap\left(R_{t} \circ F_{p}^{\prime \prime}\right)$ in $R_{t}\left(\mathbb{R}^{6}\right)$ changes continuously as $t$ goes from 0 to 1 . The orientations of the hyperplanes $R_{1}\left(\mathbb{R}^{6}\right)$ and $\mathbb{R}^{6}$ are opposite, thus the reflection $R$ changes the orientation of the intersection $F_{p}^{\prime} \cap F_{p}^{\prime \prime}$.

So we have defined an isomorphism $\Psi_{p}$ between $\operatorname{ker}\left(d F_{p}\right)$ and $\operatorname{det}\left(T \Sigma\left(F_{p}\right)\right)$ for every $p \in W^{4}$ in a compatible way (i.e., $\Psi_{p}\left|\left(U_{p} \cap U_{q}\right)=\Psi_{q}\right|\left(U_{p} \cap U_{q}\right)$ for $\left.p, q \in W^{4}\right)$. These local isomorphisms define a global isomorphism $\Psi$ between $\operatorname{ker}(d F)$ and $\operatorname{det}(T \Sigma(F))$.

\section{The invariant}

In this section we will give a geometric formula for the 3-dimensional obstruction to the existence of a regular homotopy between two immersions of $M^{3}$ into $\mathbb{R}^{5}$. This generalizes the results of 10 to immersions with non-trivial normal bundle.

Definition 5.1. Let $W^{4}$ be a compact oriented manifold with boundary $M^{3}$ and $F: W^{4} \rightarrow \mathbb{R}^{5}$ a generic map such that $f=F \mid M^{3}$ is an immersion. Recall that $\Sigma(F)$ denotes the set of singular points of $F$. Let us denote by $C(F) \subset M^{3}$ the 1-dimensional submanifold $\partial \Sigma(F)$. Choose a trivialization $\tau$ of $\operatorname{ker}(d F) \mid C(F)$ so that it points into the interior of $W^{4}$. This is possible since $f$ is non-singular (and so $\operatorname{ker}(d F)$ never lies in $\left.T M^{3}\right)$. Then $\tau$ is normal to $\Sigma(F)$ because $F$ is generic and thus $\Sigma^{1,1}(F) \cap C(F)=\emptyset$. So if we project $\tau$ into $T M$ along $\Sigma(F)$ we obtain a nowhere vanishing normal field $\nu(F)$ in $\nu\left(C(F) \subset M^{3}\right)$.

Let $U$ denote a small collar neighborhood of $C(F)$ in $\Sigma(F)$. Then clearly $U$ is orientable. Using the isomorphism $\Psi$ of Definition 4.4 the trivialization $\tau$ of 
$\operatorname{ker}(d F)$ induces an orientation of $U$. Thus $C(F) \subset \partial U$ is also oriented. So we have assigned a pair $(C(F), \nu(F))$ to $F$ as in Notation 3.1 Let $r(F)=r(C(F), \nu(F))$.

Notation 5.2. For $\chi \in H^{2}\left(M^{3} ; \mathbb{Z}\right)$ let us denote by $\operatorname{Imm}\left(M^{3}, \mathbb{R}^{5}\right)_{\chi}$ the space of immersions with normal Euler class $\chi$.

Fix a cohomology class $\chi \in H^{2}\left(M^{3} ; \mathbb{Z}\right)$. Our aim is to define an invariant $i: \pi_{0}\left(\operatorname{Imm}\left(M^{3}, \mathbb{R}^{5}\right)_{\chi}\right) \rightarrow \mathbb{Z}_{2 d(\chi)}$.

Proposition 5.3. Let $f \in \operatorname{Imm}\left(M^{3}, \mathbb{R}^{5}\right)_{\chi}$ and let $F: W^{4} \rightarrow \mathbb{R}^{5}$ be a generic map such that $\partial F=f$. Then $[C(F)]=D \chi$.

Proof. Let $\kappa$ denote an inner normal field of $M^{3}$ in $W^{4}$ that extends $\tau$ (see Definition 5.11). Then $d F \circ \kappa$ is a vector field along $f$ that is tangent to $f$ exactly at the points of $C(F)=\partial \Sigma(F)$. (If $p \in C(F)$ then the rank of $(d F)_{p}$ is 3 , moreover $d F \mid\left(T_{p} M^{3}\right)=d f$ is non-degenerate. Thus $d F\left(\kappa_{p}\right) \in d f\left(T_{p} M^{3}\right)$.) So if we project $d F \circ \kappa$ into the normal bundle of $f$ we obtain a normal field of $f$ that vanishes along $C(F)$. To see that $C(F)$ represents the normal Euler class of $f$, we have to know that it is oriented suitably.

Using the notations of Definition 5.1 we choose a function $s: W^{4} \rightarrow \mathbb{R}$ such that the derivative of $s$ in the direction of $\kappa$ (and thus $\tau$ ) is positive and $s \mid M^{3} \equiv 0$. Then there exists a collar neighborhood $V$ of $M^{3}$ in $W^{4}$ such that $F^{\prime}=(F, s) \mid V$ is an immersion. Denote by $\nu_{F^{\prime}}$ the normal bundle of $F^{\prime}$ in $\mathbb{R}^{6}$ and by $\nu_{f}$ the normal bundle of $f$ in $\mathbb{R}^{5}$. Then $\nu_{F^{\prime}} \mid M^{3}=\nu_{f}$ as oriented bundles, since $s$ is increasing along $\kappa$ (here $\mathbb{R}^{5}$ and $\mathbb{R}^{6}$ are considered with their standard orientations). By Definition 4.4 the surface of singular points $U=\Sigma(F \mid V)$ is oriented as the self-intersection of $F^{\prime}$ in $\mathbb{R}^{6}$, or more precisely, as the intersection of the zero section and a generic section of $\nu_{F^{\prime}}$. Moreover, $C(F)$ is oriented as the boundary of $U$. Thus $C(F)$ is the self-intersection of the zero section of $\nu_{F^{\prime}} \mid M^{3}=\nu_{f}$, so it is dual to the Euler class $e\left(\nu_{f}\right)=\chi$. (Here we used the naturality of the Euler class.)

Definition 5.4. Let $f \in \operatorname{Imm}\left(M^{3}, \mathbb{R}^{5}\right)_{\chi}$ and let $F: W^{4} \rightarrow \mathbb{R}^{5}$ be generic such that $\partial F=f$. Denote the algebraic number of cusps of $F$ by $\# \Sigma^{1,1}(F)$ (for the definition see [3]). Then let $j(f)=3 \sigma\left(W^{4}\right)-3 \alpha\left(M^{3}\right)+\# \Sigma^{1,1}(F)+r(F) \in \mathbb{Z}_{2 d(\chi)}$.

Note that if $\chi=0$ and $F$ is an immersion near $\partial W^{4}$ then $r(F)=0$. Thus in this case $j(f)$ agrees with the double of the invariant introduced in 10] (see Definition 2.7).

Theorem 5.5. $j(f)$ is well defined, i.e., it does not depend on the choice of the generic map $F$. Moreover, if $f_{0}$ and $f_{1}$ are regularly homotopic then $j\left(f_{0}\right)=j\left(f_{1}\right)$.

Proof. For $i \in\{0,1\}$ let $F_{i}: W_{i}^{4} \rightarrow \mathbb{R}^{5}$ be a generic map such that $\partial F_{i}=f_{i}$. Choose a regular homotopy $\left\{h_{t}: 0 \leq t \leq 1\right\}$ connecting $f_{0}$ and $f_{1}$. This defines an immersion $H: M^{3} \times I \rightarrow \mathbb{R}^{5} \times I$ by the formula $H(x, t)=\left(h_{t}(x), t\right)$. Also choose a closed collar neighborhood $U_{i}$ of $M^{3}$ in $W_{i}^{4}$ and a diffeomorphism $d_{i}: U_{i} \rightarrow$ $M^{3} \times[0, \varepsilon]$ for $i=0,1$. Let $p: M^{3} \times[0, \varepsilon] \rightarrow[0, \varepsilon]$ denote the projection onto the second factor. If $\varepsilon$ (i.e., $U_{i}$ ) is sufficiently small then $p \circ d_{i}$ is non-degenerate along $\operatorname{ker}\left(d F_{i}\right)$ for $i=0,1$ since $\operatorname{ker}\left(d F_{i}\right)$ never lies in $T M^{3}$. Let $s_{i}$ be an arbitrary smooth extension of $p \circ d_{i}$ over $W_{i}^{4}$. Now let $F_{0}^{\prime}=\left(F_{0},-s_{0}\right):-W_{0}^{4} \rightarrow \mathbb{R}^{6}$ and $F_{1}^{\prime}=\left(F_{1}, s_{1}+1\right): W_{1}^{4} \rightarrow \mathbb{R}^{6}$. Then $F_{i}^{\prime}$ is an immersion on $U_{i}$. Notice that $H \mid\left(M^{3} \times\right.$ $\{0\})=F_{0}^{\prime} \mid\left(\partial W_{0}^{4}\right)$ and $H\left|\left(M^{3} \times\{1\}\right)=F_{1}^{\prime}\right|\left(\partial W_{1}^{4}\right)$. 
Denote by $\kappa_{i}$ the inner normal field of $W_{i}^{4}$ along $M^{3}=\partial W_{i}^{4}$ and by $v_{6}$ the sixth coordinate direction in $\mathbb{R}^{6}$. Then the inner product $\left\langle d F_{0}^{\prime}\left(\kappa_{0}\right), v_{6}\right\rangle<0$ and $\left\langle d F_{1}^{\prime}\left(\kappa_{1}\right), v_{6}\right\rangle>0$. Furthermore, if $\lambda_{i}$ denotes the inner normal field of $M^{3} \times I$ along $M^{3} \times\{i\}$ for $i=0,1$ then $\left\langle d H\left(\lambda_{0}\right), v_{6}\right\rangle>0$ and $\left\langle d H\left(\lambda_{1}\right), v_{6}\right\rangle<0$. So $d H\left(\lambda_{i}\right)$ is homotopic to $-d F_{i}\left(\kappa_{i}\right)$ in the space of vector fields normal to $H \mid\left(M^{3} \times\{i\}\right)$. Using Smale's lemma there exists a regular homotopy of $H$ fixed on the boundary $M^{3} \times\{0,1\}$ that induces the above homotopy of normal fields. Denote by $H^{\prime}$ the result of this regular homotopy of $H$. Then $F_{0}^{\prime}, H^{\prime}$ and $F_{1}^{\prime}$ fit together to a smooth map $F^{\prime}$ of $W^{4}=-W_{0}^{4} \cup\left(M^{3} \times I\right) \cup W_{1}^{4}$ into $\mathbb{R}^{6}$ that is an immersion on $M^{3} \times I$. Let $\pi: \mathbb{R}^{6} \rightarrow \mathbb{R}^{5}$ denote the projection map. Then by a small perturbation of $H^{\prime}$ we can achieve that $F=\pi \circ F^{\prime}$ is generic.

Since $G=F \mid\left(M^{3} \times I\right)=\pi \circ H^{\prime}$ is prim, the singular surface $\Sigma(G)$ is oriented and a trivialization $\tau$ of $\operatorname{ker}(d G)$ is given. If we project $\tau$ into $\nu\left(\Sigma(G) \subset M^{3} \times I\right)$ we obtain a normal field $\nu$ along $\Sigma(G)$ that vanishes exactly at the cusps of $G$, i.e., where $\tau$ is tangent to $\Sigma(G)$. So $\# \Sigma^{1,1}(G)$ is equal to the algebraic number of zeroes of $\nu$, which in turn is congruent to $\operatorname{rd}\left(\left(C\left(F_{0}\right), \nu\left(F_{0}\right)\right),\left(C\left(F_{1}\right), \nu\left(F_{1}\right)\right)\right)=r\left(F_{0}\right)-r\left(F_{1}\right)$ modulo $2 d(\chi)$ by Definition 3.2 .

Now using the result of Szücs 13 that $3 \sigma\left(W^{4}\right)+\# \Sigma^{1,1}(F)=0$ we get that

$$
\begin{aligned}
-\left(3 \sigma\left(W_{0}^{4}\right)+\# \Sigma^{1,1}\left(F_{0}\right)+r\left(F_{0}\right)\right)+\left(3 \sigma\left(W_{1}^{4}\right)+\# \Sigma^{1,1}\left(F_{1}\right)+r\left(F_{1}\right)\right) & = \\
3 \sigma\left(W^{4}\right)+\# \Sigma^{1,1}\left(-F_{0} \cup G \cup F_{1}\right) & =0 .
\end{aligned}
$$

In the special case $f_{0}=f_{1}=f$ this implies that $j(f)$ is well defined, and for $f_{0}$ and $f_{1}$ arbitrary (but regularly homotopic) we get that $j$ is a regular homotopy invariant.

Proposition 5.6. For any immersion $f: M^{3} \rightarrow \mathbb{R}^{5}$ the invariant $j(f)$ is always an even element of $\mathbb{Z}_{2 d(\chi)}$, i.e., it is mapped to 0 by the epimorphism $\mathbb{Z}_{2 d(\chi)} \rightarrow \mathbb{Z}_{2}$.

Proof. Choose an immersion $f_{1} \in \operatorname{Imm}\left(M^{3}, \mathbb{R}^{5}\right)_{0}$ and denote $f$ by $f_{0}$. Since in 10 . it is proved that $j\left(f_{1}\right)$ is always even $\left(j\left(f_{1}\right)=2 i\left(f_{1}\right)\right.$ for $i$ as in Definition 2.7) it is sufficient to prove that $j\left(f_{0}\right) \equiv j\left(f_{1}\right)$ mod 2. Choose a singular Seifert surface $F_{i}: W_{i} \rightarrow \mathbb{R}^{5}$ for $f_{i}(i=0,1)$ and let $G: M^{3} \times I \rightarrow \mathbb{R}^{5}$ be a generic map such that $-F_{0} \cup G \cup F_{1}$ is a smooth map on $-W_{1} \cup\left(M^{3} \times I\right) \cup W_{2}$. Then by equation 5.1 above it is sufficient to prove that $r\left(F_{0}\right)-r\left(F_{1}\right) \equiv \# \Sigma^{1,1}(G) \bmod 2$. Since $f_{1}$ has trivial normal bundle we may choose $F_{1}$ to be an immersion in a neighborhood of $\partial W_{1}^{4}$. So $G$ is an immersion in a neighborhood of $M^{3} \times\{1\}$, moreover $r\left(F_{1}\right)=0$. The difference between the present situation and the proof of Theorem 5.5 is that now $\operatorname{ker}(d G)$ might be non-orientable. Using Definition 3.8 we get that $r_{2}\left(F_{0}\right)-r_{2}\left(F_{1}\right) \equiv$ $r\left(F_{0}\right)-r\left(F_{1}\right)$ mod 2. Let $\nu$ denote a generic normal field along $\Sigma^{1}(G)$ that extends both $\nu\left(F_{0}\right)$ and $\nu\left(F_{1}\right)$. By definition $r_{2}\left(F_{0}\right)-r_{2}\left(F_{1}\right)$ equals the mod 2 number of zeroes of $\nu$. Thus we only have to prove that $\left|\nu^{-1}(0)\right| \equiv \# \Sigma^{1,1}(G) \bmod 2$.

From now on we will denote $\Sigma^{1}(G)$ by $K$ and the line bundle $\operatorname{ker}(d G)<T\left(M^{3} \times\right.$ $I) \mid K$ by $l$. Then $l$ is tangent to $K$ exactly at the points of $\Sigma^{1,1}(G)$. For $\varepsilon>0$ sufficiently small let $\widetilde{K}$ denote the sphere bundle $S_{\varepsilon} l$. If $\varepsilon$ is sufficiently small then the exponential map of $M^{3} \times I$ defines an immersion $s: \widetilde{K} \rightarrow M^{3} \times I$ so that the double points of $s$ correspond exactly to the points of $\Sigma^{1,1}(G)$. So we have to prove that $\left|D_{2}(s)\right| \equiv\left|\nu^{-1}(0)\right| \bmod 2$. By Lemma 4.1 the surface $\widetilde{K}$ is the orientation double cover of $K$, in particular $\widetilde{K}$ is oriented and a sign can be given to each 
double point of $s$ (here we also use that $\operatorname{dim}(\widetilde{K})$ is even). The sign of a double point of $s$ is the opposite of the sign of the corresponding cusp of $G$ (since the sign of a cusp is defined as the self intersection of $K)$. Thus $\# D_{2}(s)=-\# \Sigma^{1,1}(G)$. Let $p: \widetilde{K} \rightarrow K$ denote the covering map. Then $p^{*} \nu_{K} \approx \nu_{s}$, thus $p^{*} \nu$ defines a section $\widetilde{\nu}$ of $\nu_{s}$. From the construction of $\widetilde{\nu}$ it is clear that $\# \widetilde{\nu}^{-1}(0)=2 \# \nu^{-1}(0)$.

If we perturb $s$ in the direction of $\widetilde{\nu}$ we get a self intersection point of $s$ for each element of $\widetilde{\nu}^{-1}(0)$ and two self intersection points for each double point of $s$. Thus

$$
s \cap(s+\varepsilon \widetilde{\nu})=\# \widetilde{\nu}^{-1}(0)+2 D_{2}(s)=2\left(\# \nu^{-1}(0)-\# \Sigma^{1,1}(G)\right) .
$$

So we only have to show that the left hand side is divisible by 4 .

From now on we will work in a fixed tubular neighborhood $T$ of $C\left(F_{0}\right) \subset M^{3}$. Note that $\partial(s, \widetilde{\nu})=\left(C\left(F_{0}\right)+\varepsilon \nu\left(F_{0}\right), \nu\left(F_{0}\right)\right) \cup\left(-C\left(F_{0}\right)-\varepsilon \nu\left(F_{0}\right), \nu\left(F_{0}\right)\right) \subset T \times\{0\}$. Let us denote by $C$ the one-dimensional submanifold $\left(C\left(F_{0}\right)+\varepsilon \nu\left(F_{0}\right)\right) \cup\left(-C\left(F_{0}\right)-\right.$ $\left.\varepsilon \nu\left(F_{0}\right)\right)$ of $M^{3}$. Then $s \cap(s+\varepsilon \widetilde{\nu})=r\left(C, \nu\left(F_{0}\right)\right) \in \mathbb{Z}$ since $C \sim C\left(F_{0}\right)-C\left(F_{0}\right)$ is null homologous in $M^{3}$. We define an embedding $e: C\left(F_{0}\right) \times[-\varepsilon, \varepsilon] \rightarrow T$ by the formula $e(x, t)=x+t \nu\left(F_{0}\right)$. Then $E=\operatorname{Im}(e)$ is a 2-dimensional oriented submanifold of $T$ with boundary $C$. Thus $r\left(C, \nu\left(F_{0}\right)\right)=E \cap\left(C+\nu\left(F_{0}\right)\right)$ where the right hand side is considered to be a generic intersection (each fiber of $E$ is parallel to $\left.\nu\left(F_{0}\right)\right)$. Let $n$ be a small non-zero vector field along $C\left(F_{0}\right)$ orthogonal to $\nu\left(F_{0}\right)$. Then $E \cap\left(C+\nu\left(F_{0}\right)+n\right)=\emptyset$ (this can be verified by inspecting each fiber of $T)$, thus $r\left(C, \nu\left(F_{0}\right)\right)=0$. So we get that $\# \nu^{-1}(0)=\# \Sigma^{1,1}(G)$, not just a mod 2 congruence.

Remark 5.7. A small improvement on the proof of Proposition [5.6 yields an interesting result: Let $G: M^{3} \times[0,1] \rightarrow \mathbb{R}^{5}$ be a generic map connecting the immersions $f_{0}$ and $f_{1}$. Let $K$ denote the singular surface of $G$ and let $\nu_{i}$ be a trivialization of $\operatorname{ker}(d G) \mid\left(M^{3} \times\{i\}\right)$ for $i=0,1$. Then $\# \Sigma^{1,1}(G)$ is equal to the relative twisted normal Euler class $e\left(\nu_{K} ; \nu_{0}, \nu_{1}\right)$.

Note that if $\partial K=C_{0} \cup C_{1}$ then by definition

$$
\operatorname{rd}_{2}\left(\left(C_{0}, \nu_{0}\right),\left(C_{1}, \nu_{1}\right)\right) \equiv e\left(\nu_{K} ; \nu_{0}, \nu_{1}\right) \quad \bmod 2 .
$$

If in particular $K$ is an oriented cobordism between $C_{0}$ and $C_{1}$ then

$$
\operatorname{rd}\left(\left(C_{0}, \nu_{0}\right),\left(C_{1}, \nu_{1}\right)\right)=e\left(\nu_{K} ; \nu_{0}, \nu_{1}\right)=\# \Sigma^{1,1}(G) ;
$$

here $C_{i}$ is oriented by $\nu_{i}$ using the isomorphism $\Psi$ (see Definition 4.4).

Thus $j$ may take only $d(\chi)$ different values if $d(\chi)>0$. (Since $j$ is additive if a connected sum is taken with an immersion of a sphere (Lemma 6.1) and $j(g)$ can be any even number for $g: S^{3} \rightarrow \mathbb{R}^{5}$ it follows that $j$ is an epimorphism onto $2 \mathbb{Z}_{2 d(\chi)}$.) But Theorem 2.1 implies that there are exactly $2 d(\chi)$ regular homotopy classes with normal Euler class $\chi$. So $j$ describes the regular homotopy class of $f$ only up to a $2: 1$ ambiguity. To resolve this problem we will lift the invariant $j \in \mathbb{Z}_{2 d(\chi)}$ to an invariant $I \in \mathbb{Z}_{4 d(\chi)}$. It follows from Proposition [5.6 that $I$ is always an even element, thus it defines an invariant $i \in \mathbb{Z}_{2 d(\chi)}$ by the embedding $\mathbb{Z}_{2 d(\chi)} \hookrightarrow \mathbb{Z}_{4 d(\chi)}$.

Notation 5.8. Let $f \in \operatorname{Imm}\left(M^{3}, \mathbb{R}^{5}\right)_{\chi}$ and let $F: W^{4} \rightarrow \mathbb{R}^{5}$ be a singular Seifert surface for $f$. If $\kappa$ denotes the inner normal field along $\partial W^{4}$ then let $\bar{w}(F) \in \Gamma\left(\nu_{f}\right)$ be the projection of $d F(\kappa)$ into $\nu_{f}$. If $\bar{t} \in \Gamma\left(\varepsilon_{M}^{1}\right)$ denotes a trivialization of $\varepsilon_{M}^{1}$ then we can consider $\bar{t}$ and $\bar{w}(F)$ to be sections of $\nu_{f} \oplus \varepsilon_{M}^{1}$. Let $\bar{v}(F)=\bar{w}(F)+\bar{t} \in$ $\Gamma\left(\nu_{f} \oplus \varepsilon_{M}^{1}\right)$. 
From now on we will fix a spin structure $s_{M} \in \operatorname{Spin}\left(M^{3}\right)$. If we consider $\mathbb{R}^{5}$ with its unique spin structure then for every immersion $f: M^{3} \uparrow \rightarrow \mathbb{R}^{5}$ a spin structure $s(f)$ is induced on $\nu_{f}$ by $s_{M}$. Then $s(f)$ is equivalent to a trivialization $\tau(f): \varepsilon_{M}^{3}\left|\operatorname{sk}_{2}(M) \rightarrow \nu_{f} \oplus \varepsilon_{M}^{1}\right| \operatorname{sk}_{2}\left(M^{3}\right)$ up to homotopy. Since $\pi_{2}(S O(3))=0$ the trivialization $\tau(f)$ extends to an isomorphism $\tau(f): \varepsilon_{M}^{3} \rightarrow \nu_{f} \oplus \varepsilon_{M}^{1}$, but this extension is not unique because $\pi_{3}(S O(3)) \neq 0$.

Definition 5.9. Using the above notations let $t(f), v(F) \in \Gamma\left(\varepsilon_{M}^{3}\right)$ be defined by the formulas $t(f)=\tau(f)^{-1} \circ \bar{t}$ and $v(F)=\tau(f)^{-1} \circ \bar{v}(F)$. Denote by $a(F)$ the quadruple $(C(F), \nu(F), t(f), v(F)) \in N\left(M^{3}, \chi\right)$. Then define $R(F) \in \mathbb{Z}_{4 d(\chi)}$ to be $R(a(F))$. Since the homotopy class of the map $(t(f), v(F)) \mid \operatorname{sk}_{2}\left(M^{3}\right): \operatorname{sk}_{2}\left(M^{3}\right) \rightarrow V_{2}\left(\mathbb{R}^{3}\right)$ is independent of the choice of the extension of $\tau(f)$ to $\varepsilon_{M}^{3}$ Proposition 3.14 implies that $R(F)$ is also independent of $\tau(f)$ and depends only on $s_{M}$.

Remark 5.10. Proposition 3.15 implies that $r(F) \equiv R(F) \bmod 2 d(\chi)$.

Now we can finally define a complete regular homotopy invariant.

Definition 5.11. For $f \in \operatorname{Imm}\left(M^{3}, \mathbb{R}^{5}\right)_{\chi}$ and a singular Seifert surface $F$ let $I(f) \in \mathbb{Z}_{4 d(\chi)}$ be defined as $3 \sigma\left(W^{4}\right)-3 \alpha\left(M^{3}\right)+\# \Sigma^{1,1}(F)+R(F)$. (Recall that we have fixed a spin structure $s_{M}$ on $M^{3}$ for the definition of $R(F)$.) Remark 5.10 above implies that $j(f) \equiv I(F) \bmod 2 d(\chi)$. Thus by Proposition [5.6] we get that $I(F)$ is always an even element of $\mathbb{Z}_{4 d(\chi)}$. Let us denote by $\frac{1}{2}$ the isomorphism from $2 \mathbb{Z}_{4 d(\chi)}$ to $\mathbb{Z}_{2 d(\chi)}$. Then let $i(F)=\frac{1}{2} I(F)$.

Clearly $j(f)=2 i(f)$ for every $f \in \operatorname{Imm}\left(M^{3}, \mathbb{R}^{5}\right)_{\chi}$.

Theorem 5.12. I $(f)$ is well defined, i.e., it does not depend on the choice of the generic map $F$. Moreover, if $f_{0}$ and $f_{1}$ are regularly homotopic then $I\left(f_{0}\right)=I\left(f_{1}\right)$.

Proof. Using the notations of the proof of Theorem 5.5 we only have to show that the surface $K=\Sigma(G) \subset M^{3} \times I$ satisfies Definition 3.11 I.e., there exist generic sections $t$ and $v$ of $\varepsilon_{M \times I}^{3}$ that extend $t\left(f_{i}\right)$ and $v\left(F_{i}\right)$ for $i=0,1$ and are linearly dependent exactly over $K$. The regular homotopy between $f_{0}$ and $f_{1}$ defines the immersion $H: M^{3} \times I \leftrightarrow \mathbb{R}^{5} \times I$. For $i \in\{0,1\}$ there is a canonic isomorphism $\varphi_{i}: \nu_{H} \mid\left(M^{3} \times\{i\}\right) \rightarrow \nu_{f_{i}}$. Let $\bar{w} \in \Gamma\left(\nu_{H}\right)$ denote the projection of the sixth coordinate vector $v_{6} \in \mathbb{R}^{6}$ into $\nu_{H}$. Then $\varphi_{i} \circ\left(\bar{w} \mid M^{3} \times\{i\}\right)=\bar{w}\left(F_{i}\right)$. Moreover, $K=$ $\bar{w}^{-1}(0)$ and the orientation of $K$ is defined as the self intersection of $H$ if perturbed in the direction of $\bar{w}$. Define $\bar{t}$ to be a trivialization of the $\varepsilon_{M \times I}^{1}$ component of the bundle $\nu_{H} \oplus \varepsilon_{M \times I}^{1}$ and let $\bar{v}=\bar{w}+\bar{t}$. Then $\bar{t}$ and $\bar{v}$ are linearly dependent exactly at the points of $K$. Note that $t\left(f_{i}\right)=\tau\left(f_{i}\right)^{-1} \circ\left(\varphi_{i} \oplus \operatorname{id}_{\varepsilon^{1}}\right)\left(\bar{t} \mid M^{3} \times\{i\}\right)$ and $v\left(F_{i}\right)=\tau\left(f_{i}\right)^{-1} \circ\left(\varphi_{i} \oplus \operatorname{id}_{\varepsilon^{1}}\right)\left(\bar{v} \mid M^{3} \times\{i\}\right)$. Thus we only have to define a trivialization $\tau: \varepsilon_{M \times I}^{3} \rightarrow \nu_{H} \oplus \varepsilon_{M \times I}^{1}$ such that

$$
\tau \mid\left(M^{3} \times\{i\}\right)=\varphi_{i}^{-1} \circ \tau\left(f_{i}\right) \text { for } i=0,1 .
$$

The spin structure $s_{M} \in \operatorname{Spin}\left(M^{3}\right)$ and the unique spin structure on $I$ define a spin structure on $M^{3} \times I$. Together with the unique spin structure of $\mathbb{R}^{6}$ we get a spin structure $s_{H}$ on $\nu_{H}$. When $s_{H}$ is restricted to $M^{3} \times\{i\}$ we get back the spin structure $s_{M}$. Thus $s_{H}$ defines a trivialization

$$
\tau_{H}: \varepsilon_{M \times I}^{3}\left|\operatorname{sk}_{2}\left(M^{3} \times I\right) \rightarrow\left(\nu_{H} \oplus \varepsilon_{M \times I}^{1}\right)\right| \operatorname{sk}_{2}\left(M^{3} \times I\right)
$$

satisfying equation 5.2 over the 2 -skeleton of $M^{3} \times\{0,1\}$. Note that the trivialization $\tau\left(f_{i}\right)$ is only well defined over $\operatorname{sk}_{2}\left(M^{3}\right)$ and that we can choose an arbitrary 
extension over $M^{3}$ in order to define the rotation difference. Thus we only have to extend $\tau_{H}$ to a trivialization $\tau$ of $\nu_{H} \oplus \varepsilon_{M \times I}^{1}$ and then define $\tau\left(f_{i}\right)$ by formula 5.2 .

First we extend $\tau_{H}$ to $\operatorname{sk}_{3}\left(M^{3} \times I\right) \backslash \operatorname{sk}_{3}\left(M^{3} \times\{1\}\right)$. This is possible since the obstruction to extending the trivialization over a 3 -simplex from its boundary lies in $\pi_{2}(S O(3))=0$. If $\sigma^{3}$ is a 3 -simplex of $M^{3}$ then we can extend $\tau_{H}$ to $\sigma^{3} \times I$ since it is given only on $\partial\left(\sigma^{3} \times I\right) \backslash\left(\sigma^{3} \times\{1\}\right)$. Thus we have obtained the required extension $\tau$ of $\tau_{H}$.

\section{Connected sums And COMPleteness OF The InVARIANT $i$}

Lemma 6.1. If $f \in \operatorname{Imm}\left(M^{3}, \mathbb{R}^{5}\right)_{\chi}$ and $g \in \operatorname{Imm}\left(S^{3}, \mathbb{R}^{5}\right)$ then $c(f \# g)=c(f)$, in particular $e\left(\nu_{f \# g}\right)=e\left(\nu_{f}\right)$. Moreover

$$
i(f \# g)=i(f)+(i(g) \bmod 2 d(\chi)) \in \mathbb{Z}_{2 d(\chi)} .
$$

Proof. Since $c(f)$ describes the regular homotopy class of $f \mid\left(M_{\circ}^{3}\right)$ it is trivial that $c(f \# g)=c(f)$. Let $F$ be a singular Seifert surface of $f$ and $G$ of $g$ such that $G$ is an immersion near the boundary. Then the result follows by inspecting the boundary connected sum $F \npreceq G$ and the fact that $C(G)=\emptyset$.

Theorem 6.2. Suppose that the immersions $f_{0}, f_{1} \in \operatorname{Imm}\left(M^{3}, \mathbb{R}^{5}\right)$ are regularly homotopic on $M^{3} \backslash D$, where $D \subset M^{3}$ is diffeomorphic to the closed disc $D^{3}$ (i.e., $\left.c\left(f_{0}\right)=c\left(f_{1}\right)\right)$. Then $i\left(f_{0}\right)=i\left(f_{1}\right)$ implies that $f_{0}$ is regularly homotopic to $f_{1}$.

Proof. The proof consists of two cases according to the value of $d(\chi)$.

If $d(\chi)>0$ then $i$ takes values in $\mathbb{Z}_{2 d(\chi)}$ which is a finite group. Theorem 2.1 implies that there are exactly $2 d(\chi)$ regular homotopy classes with a fixed $\mathrm{Wu}$ invariant $c$. Thus we only have to show that the invariant $i$ restricted to immersions with $\mathrm{Wu}$ invariant $c$ is an epimorphism onto $\mathbb{Z}_{2 d(\chi)}$. For this end choose an immersion $f \in \operatorname{Imm}\left(M^{3}, \mathbb{R}^{5}\right)$ such that $c(f)=c$. In [3] it is shown that $i: \operatorname{Imm}\left[S^{3}, \mathbb{R}^{5}\right] \rightarrow \mathbb{Z}$ is a bijection. Thus Lemma 6.1 implies that $c(f \# g)=c(f)=c$ for every $g \in \operatorname{Imm}\left(S^{3}, \mathbb{R}^{5}\right)$, moreover $i:\left\{f \# g: g \in \operatorname{Imm}\left(S^{3}, \mathbb{R}^{5}\right)\right\} \rightarrow \mathbb{Z}_{2 d(\chi)}$ is surjective.

If $d(\chi)=0$ then $i$ maps into $\mathbb{Z}$. Using Smale's lemma we can suppose that $f_{0}\left|\left(M^{3} \backslash D\right)=f_{1}\right|\left(M^{3} \backslash D\right)$. The normal bundles of $f_{0} \mid D$ and $f_{1} \mid D$ in $\mathbb{R}^{5}$ are trivial, choose a trivialization for both of them. Let $\tau_{0}$ be a non-zero normal field along $f_{0} \mid D$. Then $\tau_{0} \mid \partial D$ considered in the trivialization of the normal bundle of $f_{1} \mid D$ is a map $\left(\tau_{0} \mid \partial D\right): \partial D \rightarrow S^{1}$. Since $\partial D$ is homeomorphic to $S^{2}$ and $\pi_{2}\left(S^{1}\right)=0$ the normal field $\tau_{0} \mid \partial D$ can be extended to a normal field $\tau_{1}$ of $f_{1} \mid D$. Thus $\tau_{i}$ is a normal field of $f_{i} \mid D$ for $i=0,1$ and $\tau_{0}\left|\partial D=\tau_{1}\right| \partial D$.

Next choose an oriented compact manifold $W_{0}^{4}$ with boundary $M^{3}$. We push $D$ into the interior of $W_{0}^{4}$ fixing the boundary $\partial D$ to obtain a 3 -disc $D_{1} \subset W_{0}^{4}$ so that $\partial D=\partial D_{1}$ and $M_{1}^{3}=\left(M^{3} \backslash D\right) \cup D_{1}$ is a smooth submanifold of $W_{0}^{4}$. If we throw out the domain bounded by $D$ and $D_{1}$ in $W_{0}^{4}$ we obtain a 4 -dimensional submanifold $W_{1}^{4}$ of $W_{0}^{4}$ with boundary $M_{1}^{3}$. Clearly $W_{0}^{4}$ is diffeomorphic to $W_{1}^{4}$.

We can choose a generic map $F_{0}: W_{0}^{4} \rightarrow \mathbb{R}^{5}$ with the following three properties:

(1) $F_{0} \mid M^{3}=f_{0}$ and $F_{0} \mid M_{1}^{3}=f_{1}$ (where $M_{1}^{3}$ is identified with $M^{3}$ by a diffeomorphism keeping $M^{3} \backslash D$ fixed).

(2) $F_{0}$ is an immersion in a neighborhood of $D$ and $D_{1}$.

(3) If $\kappa_{0}$ denotes the inner normal field of $D$ in $W_{0}^{4}$ and $\kappa_{1}$ denotes the inner normal field of $D_{1}$ in $W_{1}^{4}$ then $d F_{0} \circ \kappa_{0}=\tau_{0}$ and $d F_{1} \circ \kappa_{1}=\tau_{1}$. 
Let $F_{1}=F_{0} \mid W_{1}^{4}$. Then (2) implies that $C\left(F_{0}\right)=C\left(F_{1}\right) \subset M^{3} \backslash D$, moreover $\nu\left(F_{0}\right)=\nu\left(F_{1}\right)$. In particular, the normal Euler class of $f_{0}$ and $f_{1}$ coincide. Thus $R\left(F_{0}\right)=R\left(F_{1}\right)$. Since $\sigma\left(W_{0}^{4}\right)=\sigma\left(W_{1}^{4}\right)$, we get that

$$
0=i\left(f_{0}\right)-i\left(f_{1}\right)=\# \Sigma^{1,1}\left(F_{0} \mid\left(W_{0}^{4} \backslash W_{1}^{4}\right)\right) .
$$

Choose diffeomorphisms $d_{0}: S_{+}^{3} \rightarrow D$ and $d_{1}: S_{+}^{3} \rightarrow D_{1}$, where $S_{+}^{3}$ denotes the northern hemisphere of $S^{3}$. Then the immersion $F_{0} \circ d_{i}$ can be extended to an immersion $f_{i}^{\prime}: S^{3} \rightarrow \mathbb{R}^{5}$ for $i=0,1$ so that $f_{0}^{\prime}\left|S_{-}^{3}=f_{1}^{\prime}\right| S_{-}^{3}$. (This is possible since $j^{1}\left(f_{0}\right)\left|\partial D=j^{1}\left(f_{1}\right)\right| \partial D$.) Now repeating the same argument as above for $f_{0}^{\prime}$ and $f_{1}^{\prime}$, we obtain that

$$
i\left(f_{0}^{\prime}\right)-i\left(f_{1}^{\prime}\right)=\# \Sigma^{1,1}\left(F_{0} \mid\left(W_{0}^{4} \backslash W_{1}^{4}\right)\right) .
$$

(Note that $\tau_{0}$ and $\tau_{1}$ have a common extension over $S_{-}^{3}$.) Thus $i\left(f_{0}^{\prime}\right)-i\left(f_{1}^{\prime}\right)=0$, so using [3] we get that $f_{0}^{\prime}$ and $f_{1}^{\prime}$ are regularly homotopic. But this implies that there exists a regular homotopy between $f_{0}^{\prime}$ and $f_{1}^{\prime}$ that is fixed on $S_{-}^{3}$ (see [7, Lemma 3.33). So $f_{0} \mid D$ and $f_{1} \mid D$ are regularly homotopic keeping the 1 -jets on the boundary fixed, which completes the proof that $f_{0}$ and $f_{1}$ are regularly homotopic.

Corollary 6.3. The map

$$
(c, i): \operatorname{Imm}\left[M^{3}, \mathbb{R}^{5}\right] \rightarrow \coprod_{c \in H^{2}\left(M^{3} ; \mathbb{Z}\right)} \mathbb{Z}_{4 d(c)}
$$

is a bijection.

We get more structure on the set of regular homotopy classes of immersions of oriented 3-manifolds into $\mathbb{R}^{5}$ if we endow it with the connected sum operation. Let us introduce the notation

$$
I(3,5)=\left\{[f]:[f] \in \operatorname{Imm}\left[M^{3}, \mathbb{R}^{5}\right] \text { for } M^{3} \text { oriented }\right\} .
$$

Then $(I(3,5), \#)$ is a semigroup whose structure is described in the following theorem.

Theorem 6.4. Let $M_{1}^{3}$ and $M_{2}^{3}$ be oriented 3-manifolds. Then

$$
H^{2}\left(M_{1}^{3} \# M_{2}^{3} ; \mathbb{Z}\right) \approx H^{2}\left(M_{1}^{3} ; \mathbb{Z}\right) \oplus H^{2}\left(M_{2}^{3} ; \mathbb{Z}\right) .
$$

If $f_{i} \in \operatorname{Imm}\left(M_{i}^{3}, \mathbb{R}^{5}\right)$ for $i=1,2$ then

$$
c\left(f_{1} \# f_{2}\right)=c\left(f_{1}\right) \oplus c\left(f_{2}\right) \in H^{2}\left(M_{1}^{3} \# M_{2}^{3} ; \mathbb{Z}\right) .
$$

Moreover, if $\chi_{i}$ denotes the normal euler class of $f_{i}$ and $\chi$ the normal euler class of $f_{1} \# f_{2}$ then $d(\chi)=\operatorname{gcd}\left(d\left(\chi_{1}\right), d\left(\chi_{2}\right)\right)$. Finally,

$$
i\left(f_{1} \# f_{2}\right)=\left(i\left(f_{1}\right) \bmod 2 d(\chi)\right)+\left(i\left(f_{2}\right) \quad \bmod 2 d(\chi)\right) \in \mathbb{Z}_{2 d(\chi)},
$$

where $i\left(f_{i}\right) \in \mathbb{Z}_{2 d\left(\chi_{i}\right)}$ for $i=1,2$.

Proof. Equation 6.1 follows from the fact that $H^{2}\left(M_{i}^{3} ; \mathbb{Z}\right) \approx H^{2}\left(M_{i}^{3} \backslash D^{3} ; \mathbb{Z}\right.$ ) (see the long exact sequence of the pair $\left(M_{i}^{3}, M_{i}^{3} \backslash D^{3}\right)$ ) and the Mayer-Vietoris exact sequence for $M_{1}^{3} \# M_{2}^{3}=\left(M_{1}^{3} \backslash D^{3}\right) \cup\left(M_{2}^{3} \backslash D^{3}\right)$.

Equation 6.2 can be seen from the description of $c\left(f_{i}\right)$ as the regular homotopy class of $f_{i} \mid \operatorname{sk}_{2}\left(M^{3}\right)$. Since $\chi=\chi_{1} \oplus \chi_{2}$ the statement about $d(\chi)$ is trivial.

Finally, equation 6.3 is obtained by taking the boundary connected sum $F_{1} \natural F_{2}$ of singular Seifert surfaces $F_{1}$ and $F_{2}$ for $f_{1}$, respectively $f_{2}$. 


\section{IMMERSIONS OF $M^{3}$ INTO $\mathbb{R}^{6}$ WITH A NORMAL FIELD}

Let $\operatorname{Imm}_{1}\left(M^{3}, \mathbb{R}^{6}\right)$ denote the space of immersions of $M^{3}$ into $\mathbb{R}^{6}$ with a normal field $\nu$. Moreover, let $\operatorname{Imm}_{1}\left[M^{3}, \mathbb{R}^{6}\right]=\pi_{0}\left(\operatorname{Imm}_{1}\left(M^{3}, \mathbb{R}^{6}\right)\right)$ be the set of regular homotopy classes of such immersions with normal fields. If we fix a trivialization of $T M^{3}$ then Hirsch's theorem [5] implies that the natural map $\operatorname{Imm}_{1}\left(M^{3}, \mathbb{R}^{6}\right) \rightarrow$ $C\left(M^{3}, V_{4}\left(\mathbb{R}^{6}\right)\right)$ is a weak homotopy equivalence.

For $f \in \operatorname{Imm}\left(M^{3}, \mathbb{R}^{5}\right)$ let $\iota(f) \in \operatorname{Imm}_{1}\left(M^{3}, \mathbb{R}^{6}\right)$ be the immersion $f$ with the constant normal field defined by the sixth coordinate vector in $\mathbb{R}^{6}$. Thus $\iota$ is an embedding of $\operatorname{Imm}\left(M^{3}, \mathbb{R}^{5}\right)$ into $\operatorname{Imm}_{1}\left(M^{3}, \mathbb{R}^{6}\right)$. As a special case of Hirsch's compression theorem we have the following proposition.

Proposition 7.1. $\iota_{*}: \operatorname{Imm}\left[M^{3}, \mathbb{R}^{5}\right] \rightarrow \operatorname{Im}_{1}\left[M^{3}, \mathbb{R}^{6}\right]$ is a bijection.

Proof. The embedding $\mathbb{R}^{5} \hookrightarrow \mathbb{R}^{6}$ induces an embedding $V_{3}\left(\mathbb{R}^{5}\right) \hookrightarrow V_{4}\left(\mathbb{R}^{6}\right)$ and thus a map $\psi:\left[M^{3}, V_{3}\left(\mathbb{R}^{5}\right)\right] \rightarrow\left[M^{3}, V_{4}\left(\mathbb{R}^{6}\right)\right]$ that makes the following diagram commutative.

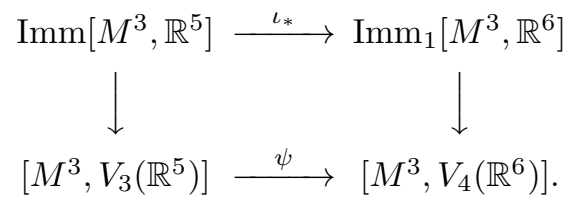

By Hirsch's theorem the vertical arrows are bijections, thus it is sufficient to prove that $\psi$ is also a bijection. To see this consider the fibration $V_{3}\left(\mathbb{R}^{5}\right) \rightarrow V_{4}\left(\mathbb{R}^{6}\right) \rightarrow$ $S^{5}$. Then from the homotopy exact sequence of this fibration we get that the homomorphism $\pi_{i}\left(V_{3}\left(\mathbb{R}^{5}\right)\right) \rightarrow \pi_{i}\left(V_{4}\left(\mathbb{R}^{6}\right)\right)$ is an isomorphism for $i \leq 3$ and this implies that $\psi$ is a bijection.

The natural forgetful map $\varphi: \operatorname{Imm}_{1}\left(M^{3}, \mathbb{R}^{6}\right) \rightarrow \operatorname{Imm}\left(M^{3}, \mathbb{R}^{6}\right)$ is a Serre fibration.

Proposition 7.2. For any immersion $f: M^{3} \rightarrow \mathbb{R}^{6}$ the normal bundle $\nu_{f}$ is trivial.

Proof. Since $M^{3}$ is spin the normal bundle $\nu_{f}$ is also spin, thus it is trivial over the 2-skeleton of $M^{3}$. Such a trivialization can be extended to the 3-simplices of $M^{3}$ because $\pi_{2}(S O(3))=0$.

So $\varphi$ is surjective and the fiber of $\varphi$ is homotopy equivalent to $\Gamma\left(\nu_{f}\right)=C\left(M^{3}, S^{2}\right)$. Thus the end of the homotopy exact sequence of $\varphi$ looks like as follows:

$$
\pi_{1}\left(\operatorname{Imm}\left(M^{3}, \mathbb{R}^{6}\right)\right) \longrightarrow\left[M^{3}, S^{2}\right] \longrightarrow \operatorname{Imm}_{1}\left[M^{3}, \mathbb{R}^{6}\right] \stackrel{\varphi_{*}}{\longrightarrow} \operatorname{Imm}\left[M^{3}, \mathbb{R}^{6}\right] \longrightarrow 0 .
$$

By Hirsch's theorem there is a bijection $\operatorname{Imm}\left[M^{3}, \mathbb{R}^{6}\right] \approx\left[M^{3}, V_{3}\left(\mathbb{R}^{6}\right)\right]$. Since $V_{3}\left(\mathbb{R}^{6}\right)$ is 2-connected and $\pi_{3}\left(V_{3}\left(\mathbb{R}^{6}\right)\right) \approx \mathbb{Z}_{2}$ we get from obstruction theory that $\left[M^{3}, V_{3}\left(\mathbb{R}^{6}\right)\right] \approx H^{3}\left(M^{3} ; \mathbb{Z}_{2}\right) \approx \mathbb{Z}_{2}$. It is well known that for $f \in \operatorname{Imm}\left(M^{3}, \mathbb{R}^{6}\right)$ the regular homotopy class of $f$ is determined by the number of its double points $D(f)$ modulo 2. This gives a geometric interpretation of the map $\varphi_{*}$ : for $(f, \nu) \in$ $\operatorname{Imm}_{1}\left(M^{3}, \mathbb{R}^{6}\right)$ the regular homotopy invariant $\varphi_{*}(f, \nu)$ is equal to $D(f)$ modulo 2 .

How can we determine the value of $\varphi_{*} \circ \iota_{*}(g)$ for a generic $g \in \operatorname{Imm}\left(M^{3}, \mathbb{R}^{5}\right)$ ? This question was answered in 12, let us recall that result now. The self-intersection set $A(g)$ of $g$ is a closed 1-dimensional submanifold of $M^{3}$ and $g(A(g))$ is also a closed 1-dimensional submanifold of $\mathbb{R}^{5}$. We say that a component $C$ of $g(A(g))$ is non-trivial if the double cover $g \mid g^{-1}(C): g^{-1}(C) \rightarrow C$ is non-trivial, i.e., if $g^{-1}(C)$ 
is connected. Let the number of non-trivial components be denoted by $\delta(g)$. In 12 Szücs proved the following.

Theorem 7.3. Suppose that $f: M^{3} \rightarrow \mathbb{R}^{6}$ is a generic immersion and that $\pi: \mathbb{R}^{6} \rightarrow$ $\mathbb{R}^{5}$ is a projection such that $g=\pi \circ f$ is also a generic immersion. Then

$$
D(f) \equiv \delta(g) \quad \bmod 2 .
$$

Note that the immersions $f$ and $g$ above are regularly homotopic in $\mathbb{R}^{6}$. Thus for any generic $g$ we have that $\varphi_{*} \circ \iota_{*}=\delta$.

Now we are going to determine the group $\pi_{1}\left(\operatorname{Imm}\left(M^{3}, \mathbb{R}^{6}\right)\right)$. Using Hirsch's theorem we get that it is isomorphic to $\pi_{1}\left(C\left(M^{3}, V_{3}\left(\mathbb{R}^{6}\right)\right)\right)=\left[S M^{3}, V_{3}\left(\mathbb{R}^{6}\right)\right]$. Here $S M^{3}$ denotes the suspension of $M^{3}$ and is a 4-dimensional CW complex. The space $V_{3}\left(\mathbb{R}^{6}\right)$ is 2 -connected and $\pi_{3}\left(V_{3}\left(\mathbb{R}^{6}\right)\right) \approx \mathbb{Z}_{2}$. Moreover, $\pi_{4}\left(V_{3}\left(\mathbb{R}^{6}\right)\right) \approx 0$. This can be seen as follows: From the homotopy exact sequence of the fibration $V_{3}\left(\mathbb{R}^{6}\right) \rightarrow$ $V_{4}\left(\mathbb{R}^{7}\right) \rightarrow S^{6}$ we get that $\pi_{4}\left(V_{3}\left(\mathbb{R}^{6}\right)\right) \approx \pi_{4}\left(V_{4}\left(\mathbb{R}^{7}\right)\right)$. It was shown by Paechter 8 that for $k \geq 4$ the isomorphism $\pi_{k}\left(V_{k}\left(\mathbb{R}^{2 k-1}\right)\right) \approx 0$ holds if $k \equiv 0 \bmod 4$. Thus obstruction theory yields that $\left[S M^{3}, V_{3}\left(\mathbb{R}^{6}\right)\right] \approx H^{3}\left(S M^{3} ; \mathbb{Z}_{2}\right) \approx H^{2}\left(M^{3} ; \mathbb{Z}_{2}\right)$.

Putting together the above results we obtain the following theorem.

Theorem 7.4. The following sequence is exact:

$$
H^{2}\left(M^{3} ; \mathbb{Z}_{2}\right) \longrightarrow\left[M^{3}, S^{2}\right] \longrightarrow \operatorname{Imm}\left[M^{3}, \mathbb{R}^{5}\right] \stackrel{\delta}{\longrightarrow} \mathbb{Z}_{2} \longrightarrow 0 .
$$

Remark 7.5. If we fix a trivialization of $T M^{3}$ then non-zero vector fields (or equivalently, oriented 2-plane fields) on $M^{3}$ correspond to maps $M^{3} \rightarrow S^{2}$. Thus the set of homotopy classes of oriented 2-plane fields on $M^{3}$ is equal to $\left[M^{3}, S^{2}\right]$ which was determined in Remark 3.3 A geometric classification of such oriented 2-plane fields, avoiding the use of a trivialization of $T M^{3}$, was carried out by Gompf (see 4, section 4). A complete set of homotopy invariants, similar to those introduced in our present paper, were obtained in 4. Gompf's result and the regular homotopy classification of immersions of $M^{3}$ into $\mathbb{R}^{5}$ are related by Theorem 7.4

\section{ACKNOWLEDGEMENTS}

I would like to take this opportunity to thank Professor András Szúcs for our long and helpful discussions and for reading earlier versions of this paper.

\section{REFERENCES}

1. Li Banghe, On classification of immersions of n-manifolds in (2n-1)-manifolds, Comment. Math. Helvetici 57 (1982), 135-144.

2. T. Ekholm, Regular homotopy and Vassiliev invariants of generic immersions $S^{k} \rightarrow \mathbb{R}^{2 k-1}$, $k \geq 4$, J. Knoth Theory Ramifications 7 (1998), no. 8, 1041-1064.

3. T. Ekholm and A. Szücs, Geometric formulas for Smale invariants of codimension two immersions, Topology 42 (2003), no. 1, 171-196.

4. R. E. Gompf, Handlebody construction of Stein surfaces, Ann. Math. 148 (1998), 619-693.

5. M. W. Hirsch, Immersions of manifolds, Trans. Am. Math. Soc. 93 (1959), 242-276.

6. J.F. Hughes and P.M. Melvin, The Smale invariant of a knot, Comment. Math. Helv. 60 (1985), 615-627.

7. A. Juhász, Regular homotopy classes of singular maps, Proc. London Math. Soc. (submitted).

8. G. F. Paechter, The groups $\pi_{r}\left(V_{n, m}\right)$, Quart. J. Math. Oxford Ser. 7 (1956), no. 2, 249-268.

9. L. Pontrjagin, A classification of mappings of the three-dimensional complex into the two dimensional sphere, Matematicheskii Sbornik 9 (1941), no. 2, 331-363.

10. O. Saeki, A. Szücs, and M. Takase, Regular homotopy classes of immersions of 3-manifolds into 5-space, Manuscripta Math. 108 (2002), 13-32. 
A GEOMETRIC CLASSIFICATION OF IMMERSIONS OF 3-MANIFOLDS INTO 5-SPACE 17

11. S. Smale, Classification of immersions of spheres in Euclidean space, Ann. Math. 69 (1959), 327-344.

12. A. Szücs, Note on double points of immersions, Manuscripta Math. 76 (1992), 251-256.

13. - On the singularities of hyperplane projections of immersions, Bull. London Math. Soc. 32 (2000), 364-374.

14. Wu, Wen-Tsün, On the immersion of $C^{\infty}$-3-manifolds in a Euclidean space, Sci. Sinica 13 (1964), 335-336.

Department of Analysis, Eötvös Loránd University, Pázmány Péter sétány 1/C, BuDAPEST, HUNGARY 1117

E-mail address: juhasz.6@dpg.hu 